\title{
Remediación de suelos contaminados con plomo (II) por fitotecnologías correctivas - Medicago sativa L. (Alfalfa)
}

pags $27-40$

Grupo de Investigación: Biotecnología

Línea de investigación: Remediación

Claudio R. Bernal B•, Lynett S. Boshell R.••

Centro de bioprospección e ingeniería química aplicada al estudio de biomoléculas e industria

Recibido: 3 de agosto de 2014

Aceptado: 5 de diciembre de 2014

\section{RESUMEN}

La concentración de plomo es variable y depende de la situación. De manera natural, el plomo presenta una concentración de 10-50 mg/kg, pero, la actividad humana aumenta en 10 a 200 veces; el plomo atmosférico se aproxima a $10 \mu \mathrm{g} / \mathrm{m} 3$ de aire; $\mathrm{Pb}$ disuelto en aguas p.e. del río Bogotá (Colombia) presenta una concentración de 49,77 KgPb/día. Medicago sativa L., se emplea como especie vegetal bioacumuladora. Los suelos del Centro Agropecuario Marengo de la Universidad Nacional de Colombia; Sede Bogotá, fueron usados para la experimentación, teniendo en cuenta que al interior de dicho Centro circula un canal de riego el cual se alimenta con aguas de la cuenca media del Rio Bogotá. Se colectaron suelos in-situ y su estudio se llevó a cabo de forma in-vitro en el Instituto Geográfico Agustín Codazzi. Un análisis factorial con dos factores y un diseño de bloques completos aleatorizados fue establecido, lo que permitió analizar la interacción de dos variables principales: $\mathrm{pH}$ y $[\mathrm{Pb}]$-incubado. Alfalfa acumula alrededor de $62 \%$ de plomo en el suelo a $\mathrm{pH}=5,5 ; 32 \%$ más en comparación con las especies de plantas (malezas) que crecen en los suelos experimentales a $\mathrm{pH}=5,4$. Alfalfa tiene la capacidad gradual de acumulación de plomo a $\mathrm{pH}=5.5$, principalmente en las hojas en comparación con tallos y raíces. Aunque los suelos experimentales se riegan con aguas del río Bogotá, éstos no pueden ser considerados con niveles excesivos de plomo que ameriten aplicar tecnologías de fito-remediación. A pesar de que se emplean aguas del rio Bogotá para el riego agrícola, no es posible afirmar alguna tendencia creciente de acumulación en los suelos; por lo menos no en los suelos experimentales.

Palabras clave: fitorremediación, metales pesados, plomo (II), fitoextracción, Medicago sativa L., Alfalfa.

\section{ABSTRACT \\ The concentration of lead varies and depends on the situation. Naturally the lead presents a concentration of $10-50 \mathrm{mg} / \mathrm{kg}$, but, activity human increases in 10 to 200 more; atmospheric lead approximates to $10 \mu \mathrm{g} /$ m3 of air; lead dissolved in water Bogotá river (Co- lombian) a concentration of $49,77 \mathrm{KgPb} /$ day. Is used}

- Docente investigador, Ingeniería Química, Fundación Universidad de América. Claudio.bernal@investigadores.uamerica.edu.co

•• Estudiantes coinvestigadores, programa de ingeniería química. 
as a plant species acumulation a Medicago Sativa L. The soils of the Agricultural Center Marengo the Universidad Nacional of Colombia; Bogota headquarters, were used for experimentation, taking into account that said inside Center circulates an irrigation canal which is fed with water from the middle of the Bogotá River basin. Collection of soils in situ, and his study was carried out in vitro in the Agustín Codazzi Geographical Institute. A factor with two factors analysis and a complete block design randomized was established, allowing you to analyze the interaction of two main variables: $\mathrm{pH}$ and $[\mathrm{Pb}]$. Alfalfa builds up around $62 \%$ of lead in the soil at $\mathrm{pH}=5.5 ; 32 \%$ more compared with species of plants (weeds) that grow in the experimental soils at $\mathrm{pH}=5.4$. Alfalfa has the ability to gradual accumulation of lead at $\mathrm{pH}=5.5$, mainly in the leaves compared to stems and roots. Although the experimental soils are irrigated with water from the Bogota River, these cannot be considered with excessive levels of lead which warrant applied phyto-remediation technologies. While using the Bogotá River for agricultural irrigation, it is not possible to affirm a growing trend of accumulation in soils; at least not in the experimental soils.

Key words: fitoremediation, heavy metals, lead (II), fitoextraction, Medicago Sativa L. Alfalfa.

\section{INTRODUCCIÓN}

Es de nuestro interés caracterizar, y al mismo tiempo proponer soluciones tecnológicas de biorremediación en suelos colombianos, principalmente en aquellos que son y/o han sido regados con aguas provenientes de ríos con un alto grado de contaminación y más aún cuando dichos suelos son empleados en labores agrícolas, pecuarias $u$ otras relacionadas y que implican impacto en la salud humana. La caracterización y/o la propuesta de solución biorremediadora estuvieron dirigidas a evaluar la capacidad de alfalfa (Medicago sativa L.) como especie bioacumuladora de plomo $(\mathrm{Pb})$; según Baker y Brooks (1989), y según Ebbs et al., (1997) establecer si esta especie vegetal posee la característica de especie vegetal biorremediadora ideal.
En investigaciones realizadas sobre suelos de la cuenca del Rio Bogotá (puntos de muestreo a lo largo de la cuenca) se ha encontrado diferentes estados de contaminación por diversos metales pesados. Ruiz, J. (2011) determinó una concentración inicial de cadmio $(\mathrm{Cd})$ de $3.97 \mathrm{mg} / \mathrm{kg}$. En la misma investigación, y en vástago fresco de lechuga, se obtuvo concentraciones promedio de cadmio de $0.45745 \mathrm{mg} /$ kg (rango: 0.2558 y 0.6010$)$. En otros estudios, Acosta, M. y Montilla, J. (2011) analizando los mismos puntos de muestreo del rio, encontraron valores promedio de cadmio de $3.75 \mathrm{mg} / \mathrm{kg}$ (rango $2.0-4.75$ ) y de plomo $15.81 \mathrm{mg} / \mathrm{kg}$ (rango 12.0-16.75). Los valores indicados son muy superiores a los niveles máximos tolerables de cadmio para hortalizas: $0.05 \mathrm{mg} / \mathrm{kg}$ (Comisión del Codex Alimentaruis - CX/FAC 99/21) y para $\mathrm{Pb}$ de $0.1 \mathrm{ppm}$ (Legislación Europea).

$\mathrm{El} \mathrm{Pb}$ no posee ninguna función metabólica específica para los seres vivos (Abollino et al., 2002; García y Dorronsoro, 2005), por el contrario, su ingestión; inclusive en dosis bajas, afecta de forma negativa casi todos los órganos y sistemas de los seres vivos (Matte, 2003). Por otra parte, el $\mathrm{Pb}$ puede incorporarse a los suelos como un componente natural y/o causado por actividades antropogénicas y una vez en el suelo puede quedar retenido en dicha estructura. También puede ser movilizado en la solución del suelo mediante diferentes mecanismos biológicos y químicos (Pagnanelli et al., 2004). De los mecanismos biológicos y químicos, aunque se desconocen a profundidad, el $\mathrm{Pb}$ es absorbido por plantas (Angelova et al., 2004) como primer paso para la entrada de éste en la cadena alimentaria (Lannacone y Gutierrez, 1999; Arkhipchuk et al., 2000). La acumulación o hiperacumulación Brooks et al., (1977) de plomo en especies de plantas, o biorremediación por fitoextracción se define como la cantidad de la sustancia que permanece en dicho organismo después de un tiempo en exposición (Depledge et al., 1994), para ello, investigaciones realizadas por Chen, (1992); Wang et al., (1994), Chen et al., (1996) establecieron los valores, para plo$\mathrm{mo}$, de: $\mathbf{A}_{[\mathrm{Pb}]}=\mathbf{5 0}$ (definidos como el límite inferior 
LÍNEA DE INVESTIGACIÓN: REMEDIACIÓN

de concentración frecuente de metales pesados encontrados en los suelos); $\mathbf{B}_{[\mathrm{Pb}]} \mathbf{= 3 0 0}$ (definidos como el nivel aceptable de metales pesados en los suelos), y $\mathbf{C}_{[\mathrm{Pb}]}=\mathbf{5 0 0}$ (niveles excesivos en muy altas concentraciones de metales que indican la necesaria intervención).

Las técnicas de fitoextracción presentan limitaciones ejempladas en el "efecto barrera" de cada especie vegetal. Las plantas pueden acumular un determinado elemento solamente hasta una determinada concentración. Otras especies de plantas fundamentan su acumulación de $x$ o $y$ metal en la parte aérea en formas notóxicas. A partir de estos hechos las especies de plantas se han clasificado en cuatro categorías, a saber: 1) Sin efecto barrera, las que concentran el elemento químico ( $x$ o y metal) sin restricciones y de forma continua; 2) Efecto semi-barrera, que concentran entre 30 y 300 veces la concentración del metal considerada una concentración normal en la planta; 3) Con efecto-barrera, de hasta 3-30 veces la concentración del elemento considerada normal en la planta; $y$, 4) Con efecto-barrera de fondo, que no superan las concentraciones del elemento en determinada especie de planta (UCLM, 2007). (A)

Alfalfa se caracteriza por fijar nitrógeno y fósforo en el suelo, es tolerante a bajas temperaturas de 10 a $15^{\circ} \mathrm{C}$ bajo cero y temperaturas medias anuales de $15^{\circ} \mathrm{C}$, es una especie de planta tolerante a la sequía, pero muy susceptible a excesos de agua y a suelos ácidos por debajo de pH 6,4 (Guerrero, 1999). Niu et al., (2007) al comparar la acumulación de cadmio y plomo en alfalfa encuentran que esta especie acumula 0,711 de cadmio y 0,888 de plomo. En diferentes estudios se señala que alfalfa es utilizada en fito-remediación de compuestos aromáticos, Yongqing et al., (2005), de metales pesados, Niu et al., (2007);

Caracterizamos tanto química, como física y biológica los suelos del CAM de forma invitro. En esta investigación se consideró como variable principal la acumulación de plomo en tejido vegetal, ya que integra tanto el grado de absorción del metal por Medicago sativa L., así como el efecto negativo que las concentraciones excesivas del metal que puede tener sobre la producción de materia seca. Con esta variable se puede evaluar el potencial efectivo de esta especie de planta para remediación. Dicho potencial se calculó en hojas, tallos, raíz y en la totalidad de la biomasa colectada.

\section{METODOLOGÍA}

\section{Materiales}

Materiales, Reactivos, Equipos y Suministros

Materiales:

- Muestras de suelos, tomadas in-situ, en los lotes 15 y 16 del CAM.

- Especies Vegetales: Panicum (Panicum clandestinum); Diente de león (Taraxacum officinale) y Rumex (Rumex crispus) localizadas en los lotes 15 y 16 del CAM. Alfalfa (Medicago sativa L.), a partir de semillas que fueron adquiridas en mercados de semillas-Bogotá.

\section{Reactivos:}

- Para preparación (acidez) de incubación de suelos: $\left[\mathrm{HNO}_{3}\right]=65 \%$ para $\mathrm{pH}=5,5$ a 4,5 , y para mover el pH de 5,5 a 6,5 se usa el encalado con $\mathrm{CaCO}_{3}(\mathrm{~s})$

- Para incubación de suelos: $\mathrm{Pb}\left(\mathrm{NO}_{3}\right)_{2}$.

\section{Equipos}

- Medición de índices de retención de humedad: banco de pruebas - IGAC.

- Molienda de muestras de tejido vegetal: Molino de Aspas - Arthur Thomas \& CO - Instituto Geográfico Agustín Codazzi (IGAC).

- Molienda de sólidos-suelos: Molino APOLO 
- Digestión de Muestras de tejido vegetal: Digestor MicroOndas - Milestone 1200.

- Cuantificación: Espectrofotómetro de Absorción Atómica.

- Otros: Potenciómetros pH-METER.

Suministros

- Implementos de laboratorio (vidrio) - IGAC. La mayor parte de implementos están sujetos a los diferentes métodos de medición de pruebas.

\section{MÉTODOS}

La presente investigación se realizó en el Centro Agropecuario Marengo (CAM) de la Universidad Nacional de Colombia; localizado en el Municipo de Mosquera-Cundinmarca (Colombia). Las semillas de alfalfa fueron acondicionadas a la temperatura y humedad del laboratorio-IGAC y de acuerdo a los datos técnicos de etiquetas comerciales. De acuerdo al arreglo experimental, llustración 1, resultaron 18 experimentos los cuales se realizaron por triplicado, para un total de 54; donde cada uno de éstos fue considerado como una unidad experimental (UE). Una UE contiene un kilogramo de suelo del CAM que fue colectado in-situ, pero tratado in-vitro en el IGAC; el cual fue previamente caracterizado a qué tipo de suelo corresponde. Las muestras se depositaron en recipientes para los tratamientos respectivos y controles futuros. El tratamiento de cada UE consistió en un pre-tratamiento de la muestra de suelos: secado, molienda, tamizado en tamiz $D p \leq 2 \mathrm{~mm}$. Seguidamente se realiza un tratamiento por tres semanas con $\mathrm{HNO}_{3}$ y con $\mathrm{CaCO}_{3}$, se continua con la incubación con plomo durante 8 semanas - en forma de $\left.\mathrm{Pb}\left(\mathrm{NO}_{3}\right)_{2}\right)$. Finalmente, se procede a la siembra de las semillas de alfalfa; 50 semillas por UE.

\section{Desarrollo experimental}

Dos variables y/o factores principales se analizaron: Potencial de hidrogeniones $(\mathrm{pH})$ y concentración de plomo (II) incubado $\left[\mathrm{Pb}_{\|}\right]$; que fueron codificados de la siguiente manera: $\mathrm{pH}_{\mathrm{sML} 15}$ y $[\mathrm{Pb}]_{\mathrm{SML} 15}$. (sML15: suelos de Marengo - Lote 15).

Para el factor principal, $\mathrm{pH}_{\mathrm{SML} 15}$ se determinaron 3 niveles: $4.5 ; 5.5$ y 6.5. Para $[\mathrm{Pb}]_{\text {sML15incubación }} 6$ niveles: [0], [5], [20], [80], [160] y [320] ( $\mathrm{mgPb} / \mathrm{kg}_{\mathrm{sM}}$ en forma de $\left.\left(\mathrm{Pb}\left(\mathrm{NO}_{3}\right)_{2}\right)\right)$. En la etapa de pre-experimentación fue determinada la concentración inicial $[\mathrm{Pb}]_{\mathrm{SML} 15 \text { inicial }}=25,40 \mathrm{mgPb} / \mathrm{kg}$. Lo anterior sugirió que los 6 niveles de $[\mathrm{Pb}]_{\text {sMinL15icubación }}$ se modifiquen de la siguiente manera: [25.40], [30.40], [45.40], [125.40], [185.40] y [345.40]. Como variable respuesta fue establecida la biodisponibilidad, en $[\mathrm{Pb}]_{\text {tejidosffalfa }}$. Bernal, BCR. (2008)

\section{Ilustración 1. Arreglo experimental}

\begin{tabular}{|c|c|c|c|c|c|c|}
\hline & \multicolumn{6}{|c|}{ Incubación de plomo en mgPg/kg muestra (MCCn) } \\
\hline Bloque & $0(25.40)$ & $5(30,40)$ & $20(45,40)$ & $80(105,40)$ & $160(185,40)$ & $320(345,40)$ \\
\hline 18UEB1 $\mathrm{pH}^{4.5}$ & $\mathrm{MCC}_{1-4}$ & $\mathrm{MCC}_{5-6}$ & $\mathrm{MCC}_{7-8}$ & $\mathrm{MCC}_{10-12}$ & $\mathrm{MCC}_{13-15}$ & $\mathrm{MCC}_{16-18}$ \\
\hline 18UEB2pH ${ }^{5.5}$ & $\mathrm{MCC}_{19-21}$ & $\mathrm{MCC}_{22-24}$ & $\mathrm{MCC}_{25-27}$ & $\mathrm{MCC}_{28-30}$ & $\mathrm{MCC}_{31-33}$ & $\mathrm{MCC}_{34-36}$ \\
\hline 18UEB3pH ${ }^{6.5}$ & $\mathrm{MCC}_{27-30}$ & $\mathrm{MCC}_{40-42}$ & $\mathrm{MCC}_{43-45}$ & $\mathrm{MCC}_{46-48}$ & $\mathrm{MCC}_{49-51}$ & $\mathrm{MCC}_{52-5}$ \\
\hline
\end{tabular}

La biomasa generada en cada MCC se divide en tres fracciones: hojas, tallo y raíz con el fin de analizar y observar en qué lugar de la planta tiende a acumularse de mayor forma las concentraciones de plomo incubadas en el suelo de acuerdo al pH.

\begin{tabular}{|l|l|l|l|}
\hline Bloque & $0(25,40)$ & \multicolumn{2}{|l}{} \\
\hline $18 U E / B_{1} 1 \mathrm{pH}^{4.5}$ & MCC $_{1-3}$ & \multicolumn{2}{|l}{} \\
\cline { 1 - 2 } & MCC $_{1}$ & Tallo & Raíz \\
\cline { 2 - 3 } & Hojas &
\end{tabular}


LÍNEA DE INVESTIGACIÓN: REMEDIACIÓN

\section{Incubación de plomo y pH}

Se definieron tres bloques (llustración 1): 18UE/Bloque que corresponden a cada uno de los niveles de la variable $[\mathrm{Pb}]$ vs. Factor $\mathrm{pH}$ : 4.5; 5.5 y 6.5 , obteniendo: $18 \mathrm{UE} / \mathrm{B}_{1} \mathrm{pH}^{4.5} ; 18 \mathrm{UE} /$ $\mathrm{B} 2 \mathrm{pH}^{5.5}$ y $18 \mathrm{UE} / \mathrm{B} \mathrm{pH}^{6.5}$. Corresponde a un arreglo factorial con dos factores, bajo un diseño de bloques completamente aleatorizados.

Durante el cultivo de la alfalfa en las UE se controla la humedad del suelo y se sigue la evolución de las plantas de alfalfa con respecto a su altura y follaje. Finalmente, se lleva a cabo un proceso que consiste en fraccionar la planta de acuerdo a raíz, tallos, hojas, se procede a una etapa de secado y molienda en un molino de Aspas marca "Arthur Thomas \& CO.", por un tamaño de partícula $\leq 2 \mathrm{~mm}$ para su fácil manipulación cuando se deba llevar a análisis químico. Después de moler la muestra de tejido vegetal se pone la muestra en frascos de vidrio dentro de un horno a $70^{\circ} \mathrm{C}$ para terminar de eliminar la humedad que quede en la muestra y llevarla a análisis de cuantificación. Se continúa con un proceso de digestión ácida y se lleva a lectura-cuantificación por Espectrofotometría de Absorción Atómica.

\section{Caracterización de materiales}

Se realizaron tres determinaciones básicas: 1) la concentración $\left([\mathrm{Pb}]_{\text {inicial }} ;[\mathrm{Pb}]_{\text {residual }}\right)$ en las UE. 2) Acumulación de plomo en tejidos (raíz, tallos, hojas) de Alfalfa y 3) se analizó el material vegetal que se encontró como malezas. Los análisis siguieron los protocolos del Instituto Geográfico Agustín Codazzi (IGAC): análisis químicos: 1) capacidad de cambio catiónico; 2) bases intercambiables; 3) fósforo disponible en suelos; 4) carbón orgánico; 5) textura de suelo; 6) $\mathrm{pH}$ de suelos; 7) Valoración de $\mathrm{Cu}, \mathrm{Fe}, \mathrm{Mn}$ y Zn disponibles; 8) Boro disponible en suelos; 9) Nitrógeno total; 10) cuantificación de plomo total; 11) valoración de sulfatos; 12) valoración de los cloruros en extractos de suelos; 13) valoración de bicarbonatos y carbonatos en extractos de suelos. Análisis físicos: 1) retención de humedad. Análisis biológicos: 1) tinción de hongos;
2) inoculación de medios de cultivo para hongos y bacterias; 3) inoculación de medio de cultivo para microorganismos fijadores de nitrógeno. Análisis foliar: 1) medición de $\mathrm{N}, \mathrm{P}, \mathrm{K}, \mathrm{Ca}, \mathrm{Mg}$, $\mathrm{Mn}, \mathrm{Fe}, \mathrm{Zn}, \mathrm{Cu}$, y B; 2) Azufre en tejido vegetal

\section{RESULTADOS}

El análisis químico de los suelos del CAM se detallan en el cuadro I. Estos valores representan la característica de cada una de las UE. Con respecto al análisis foliar de especies de plantas; consideradas como maleza, se muestran en el Cuadro II. Lo naterior permite establecer algunas relaciones entre las especies cultivadas; y que se encuentran como maleza, con las características de los suelos del CAM. (Boshell y Bernal, 2008)

En la Figura 1 se indica la acumulación de plomo en tejido vegetal de plántulas de alfalfa de acuerdo al pH e incubación. Se hizo mediante una comparación de los porcentajes de plomo acumulado en la parte aérea (hojas), tallos y raíz de alfalfa, con respecto a la acumulación total.

En la figura 3 se indica el plomo residual en suelos del CAM. La concentración de plomo inicial, $[\mathrm{pb}]_{\text {inicial }}$ fue determinada como $25,40 \mathrm{mgPb} /$ $\mathrm{kg}_{\mathrm{sM}}$ a un $\mathrm{pH}_{\text {inicial }}$ de 5.4. Luego de la remediación, alfalfa acumula (\%) a pH:4,5=46.83 $-\mathrm{SD}=14.83$; a $\mathrm{pH}: 5,5=47.15-\mathrm{SD}=13.9$, y a $\mathrm{pH}: 6.5=49.26$ $\mathrm{SD}=6.88$. El plomo residual $(\%)$ a $\mathrm{pH}: 4,5=38.88$; $\mathrm{pH}: 5,5=45.62$ y a $\mathrm{pH}: 6,5=38.05$.

En la Figura 2 se observa la fitodisponibilidad de plomo en alfalfa de acuerdo al $\mathrm{pH}$ y la concentración (incubación) de plomo en las UE.

\section{DISCUSIÓN}

Los niveles registrados de potasio/fosforo/ nitrigeno: K-P-N (elementos fertilizantes) son indicadores del nivel favorable de fertilización, lo que ha permitido que se produzca la capa 

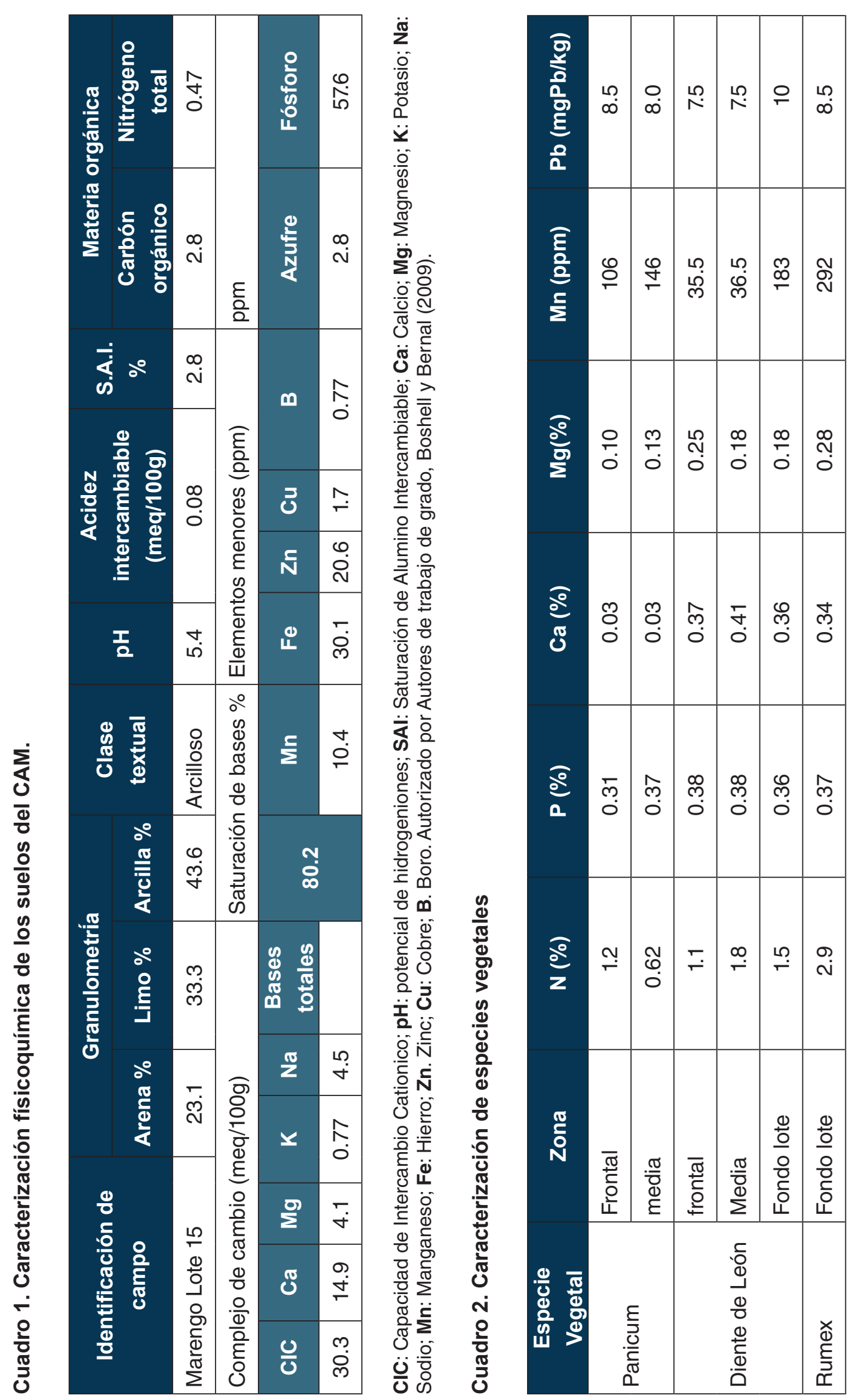

ช⿺

$\frac{0}{\mathrm{O}} \overline{\overline{0}}$

है कू

造 :

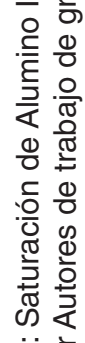

후을

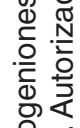

을 음

$\frac{\pi}{\frac{\pi}{0}} \frac{0}{0}$

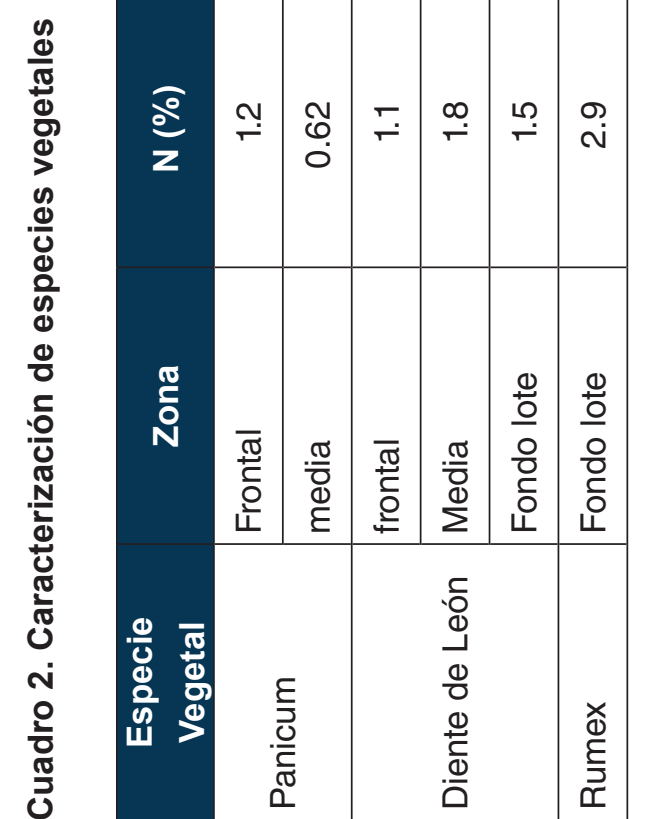

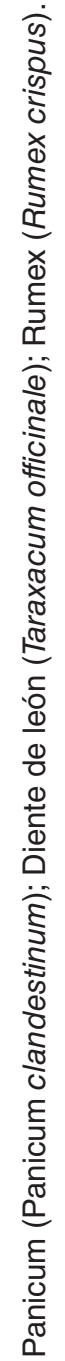


LÍNEA DE INVESTIGACIÓN: REMEDIACIÓN

Figura 1. Distribución de la concentración de Plomo en diferentes parte de alfalfa, según tratamientos de $\mathrm{pH}$ e incubación (En mg Pb/kg).

Partes de la planta de alfalfa: Hojas — Tallo — Raiz
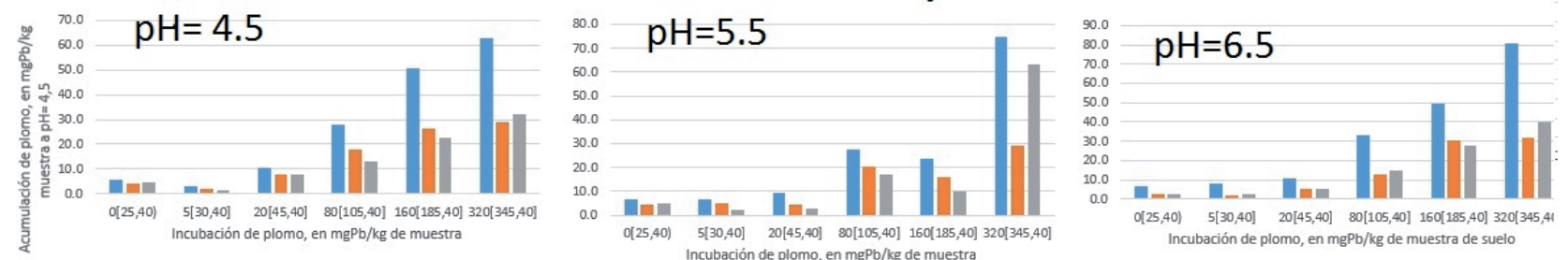

Incubación de $\mathrm{Pb}$ en $\mathrm{mgPb} / \mathrm{kg}$

Figura 2. Efecto del pH sobre la biodisponibilidad.

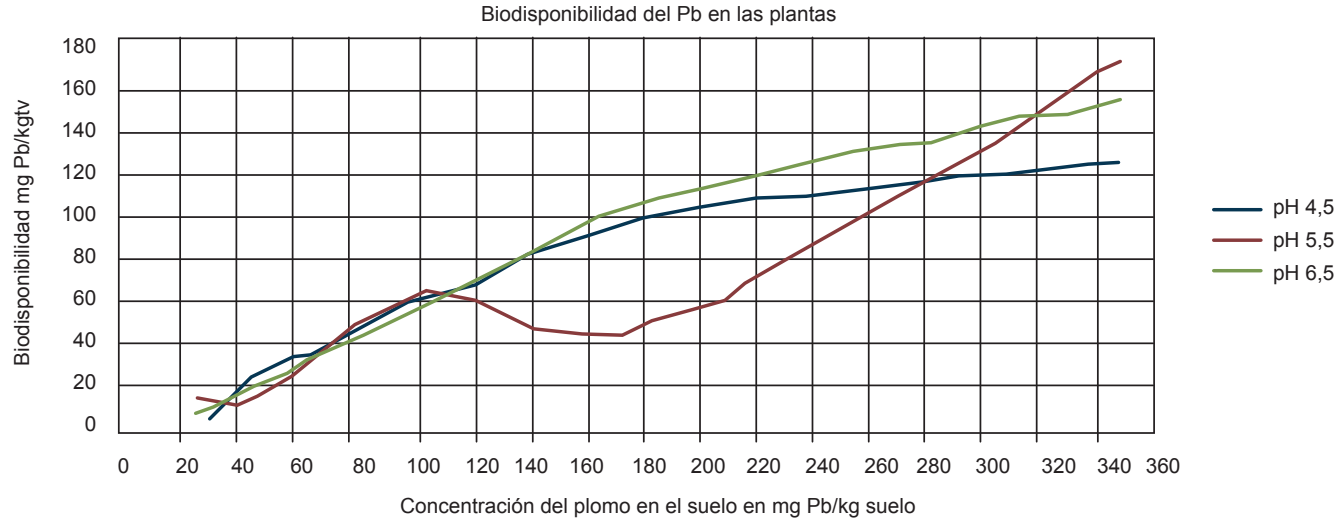

Concentración de $\mathrm{Pb}$ en el suelo en $\mathrm{mgPb} / \mathrm{kg}$

Figura 3. Plomo residual en suelos del CAM, de acuerdo a los tratamientos: pH e Incubación.

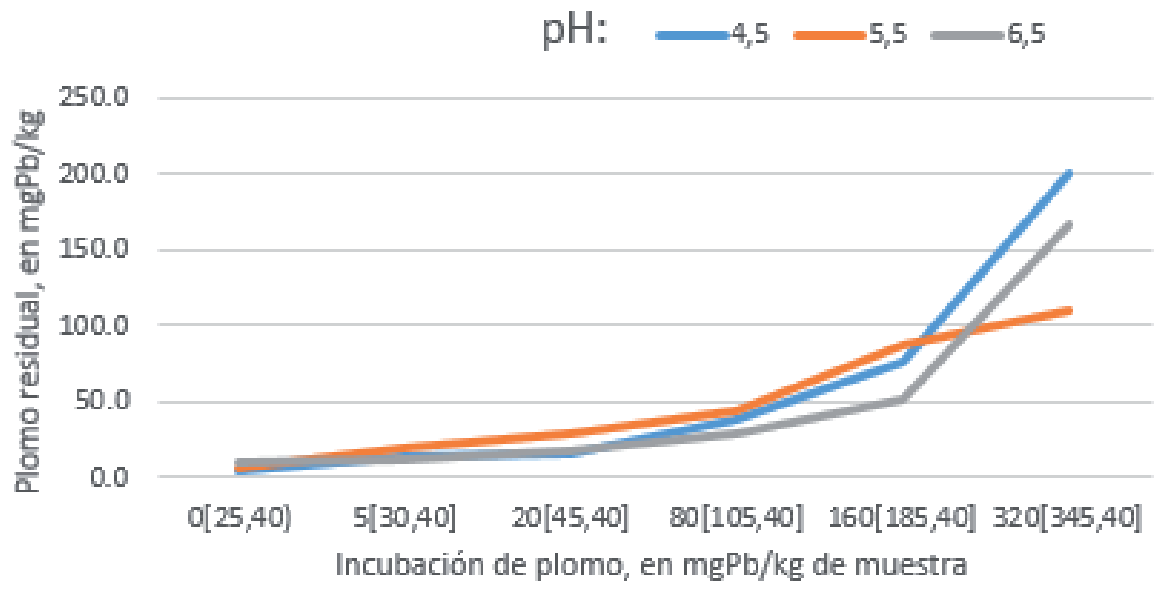

Incubación de plomo en mgPb/kg 
vegetal en dichos suelos. Con respecto a las bases Intercambiables (Ca/Mg; $\mathrm{Mg} / \mathrm{K} ; \mathrm{Ca} / \mathrm{K}$; $\mathrm{Ca}-\mathrm{Mg} / \mathrm{K}$ ) en conjunto, y de manera general, muestran relaciones ideales y buena interacción, con alguna debilidad en la relación $\mathrm{Ca} / \mathrm{K}$, lo que indicaría que el suelo investigado no necesitaría ninguna enmienda, ni fertilizantes $\mathrm{y}$ fomentaría buenas funciones físicoquímicas $\mathrm{y}$ de soporte a la capa vegetal existente y/o más adelante la especie vegetal bioremediadora. Las bases totales se conocen como la sumatoria de los valores de la capacidad de intercambio catiónico (CIC), es un valor considerado alto: 30.3 mequivalentes $/ 100 \mathrm{~g}$, lo que indica que este suelo sea clasificado como arcilloso - suele retener y liberar una mayor cantidad de cationes.

Se observa una tendencia al crecimiento a medida que aumenta la concentración de $\mathrm{Pb}$ y $\mathrm{pH}$ durante su incubación. Sin embargo, dicho crecimiento es menor cuando el $\mathrm{pH}=4.5$; respecto a los valores de $\mathrm{pH} 5.6$ y 6.5 , lo que indicaría cierta inhibición al crecimiento a $\mathrm{pH}$ ácido $\mathrm{y}$, a $\mathrm{pH}$ con tendencia a un estado alcalino, podría favorecer el crecimiento, pero sin sobrepasar el valor óptimo de $\mathrm{pH}=7.2$. Luego de cumplir con el ciclo de 45 días de cultivo se procede a realizar la cosecha de las plántulas, seguidamente secar, moler, tamizar y empacar el material-biomasa.

Biomasa seca generada para los distintos bloques (gramos): $18 \mathrm{UE} / \mathrm{B}_{1} \mathrm{pH}^{4.5}=(78.2)$ con un contenido de $329.9 \mu \mathrm{gPb} / \mathrm{g}_{\text {muestra vegetal; }}$ para $18 \mathrm{UE} / \mathrm{B}_{1} \mathrm{pH}^{5.5}(158.5)$ y $328.7 \mu \mathrm{gPb} / \mathrm{g}_{\text {muestra vegetal }}$ $\mathrm{y}$, para un $18 \mathrm{UE} / \mathrm{B} \mathrm{pH}^{6.5}$ (176.6) y $366.8 \mathrm{\mu gPb} /$ $g_{\text {muestra vegetal }}$

Sobre la distribución de plomo en alfalfa, se determinaron diferencias significativas en la distribución de $\mathrm{Pb}$ en alfalfa. Como se puede apreciar en la Figura 1, la parte (azul-hojas) es la parte que mayormente acumula plomo en cada uno de los tratamientos ( $\mathrm{pH}$ e incubación), seguido de tallo (naranja) y raíz (gris). En los estudios estadísticos, y para tallo-raíz, no muestran diferencia significativa en cuanto a la acumula- ción de plomo, con excepción de $\mathrm{pH}=5.5$ que muestra una acumulación en raíz.

Nuestros resultados mostraron que los suelos del CAM presentan un grado moderado de contaminación con plomo, entre $300-400 \mathrm{mgPb} /$ $\mathrm{kg}_{\mathrm{sM}}$. La Agencia de Protección al Ambiente (EPA) (por sus siglas en Inglés: Environmental Protection Agency) justifica intervenir los suelos mediante remediación cuando la concentración de $\mathrm{Pb}$ en suelos excede de $300-500 \mathrm{mgPb} / \mathrm{kg}$; caso de uso residencial, o $2000 \mathrm{mgPb} / \mathrm{kg}$ de muestra de suelo para uso industrial.

A partir de los datos encontrados se observa que se produce mayor biomasa cuando incrementa el $\mathrm{pH}$, pero alfalfa no se adapta a un $\mathrm{pH}$ mayormente ácido, tal y como lo muestra Infoagro, 2007: "el factor limitante en el cultivo de alfalfa es la acidez, excepto en la germinación; pudiéndose ser hasta $\mathrm{pH}=4$ " y que seguidamente lo comenta Molina, (1998) cuando manifiesta que un pH ácido ocasiona reducción de crecimiento de las plantas, reducción de la disponibilidad de algunos elementos nutricionales tales como el calcio $(\mathrm{Ca})$, magnesio $(\mathrm{Mg})$, potasio $(\mathrm{K})$, fósforo $(\mathrm{P})$, y favorece la proliferación de elementos tóxicos para las plantas como aluminio (Al) y manganeso $(\mathrm{Mn})$. Los valores de $\mathrm{pH}$ de 5.5 y 6.5 son valores que están por debajo del valor óptimo de cultivo ( $\mathrm{pH}=7.2)$, lo que contribuye, por encalamiento, a incrementar la fitodisponibilidad de la cantidad de iones de calcio para esta especie vegetal $y$, por ende, a reducir la absorción de Al y Mn.

\section{Capacidad hiperacumuladora}

Por definición, cuando se encuentra concentraciones de metales pesados en $x$ o y especies vegetales equivalentes o mayores a $1000 \mu \mathrm{gPb} /$ gmv ( $0.1 \%$ de peso seco), éstas especies se denominan hiperacumuladoras de plomo Baker y Brooks, 1989. Alfalfa acumula plomo entre 330 a $367 \mu \mathrm{gPb} / \mathrm{g}_{\mathrm{sM}}(0.1 \%$ de peso seco). Si la Biomasa seca generada para los distintos bloques (gramos): $18 \mathrm{UE} / \mathrm{B} 1 \mathrm{pH}^{4.5}=(78.2)$ con un contenido de $329.9 \mu \mathrm{gPb} / \mathrm{g}_{\text {muestra vegetal }}$; para $18 \mathrm{UE} /$ 
$\mathrm{B} 1 \mathrm{pH}^{5.5}(158.5)$ y $328.7 \mu \mathrm{gPb} / \mathrm{g}_{\text {muestra vegetal }} \mathrm{y}$, para un $18 \mathrm{UE} / \mathrm{B} 1 \mathrm{pH}^{6.5}(176.6)$ y $366.8 \mu \mathrm{gPb} / \mathrm{g}_{\text {muestra }}$ vegetal., indicaría que alfalfa no se podría definir como una planta hiperacumuladora, pero, y por un lado, Gardea-Torresdey et al., (....) indica que es una especie hiperacumuladora en $99.6 \%$, con lo que estariamos de acuerdo y si se tiene en cuenta los estudios de Bonilla-Valencia, 2013 donde encuentran que al aumentar la concentración (incubación) de suelos experimentales en los primeros 30 días, Medicago Sativa L., aumenta la concentración de plomo, pero a los 90 días se observa una reducción de este metal.

Nuestros resultados mostraron que los suelos del CAM presentan un grado moderado de contaminación con plomo, entre $300-400$ mgPb/ $\mathrm{kg}_{\mathrm{sM}}$. La Agencia de Protección al Ambiente (EPA) (por sus siglas en Inglés: Environmental Protection Agency) justifica intervenir los suelos mediante remediación cuando la concentración de $\mathrm{Pb}$ en suelos excede de $300-500 \mathrm{mgPb} / \mathrm{kg}$; caso de uso residencial, o $2000 \mathrm{mgPb} / \mathrm{kg}$ de muestra de suelo para uso industrial.

Al ser un suelo de tipo arcilloso (cargado negativamente) facilita el intercambio con los cationes. Por otra parte, los requerimientos del suelo en N-P-K muestran niveles aceptables respecto a sustancias fertiizantes. Con respecto al carbón orgánico $=2,8 \%$, este valor indica que los suelos son bajos en contenido de materia orgánica.. La textura, \% de arena en suelos $=23,11 \%$; porcentaje de arcilla en suelos: $43,638 \%$ y porcentaje de limo $=33,25 . \mathrm{pH}=5,4$ - éste suelo es fuertemente ácido, que según el IGAC es común estos suelos en Colombia. La ácidez intercambiable $=0,08 \mathrm{meq} / 100$, situación que está relacionada con el complejo de intercambio por la adsorción electrostácia expresada en los sitios de intercambio cationico presentes en el suelo. El \% de saturación de Aluminio $(\% \mathrm{SAI})=0,3 \%$. Este valor se interpreta según las guías como un suelo sin problemas en general, indicando que este tipo de suelo no tien problemas de contaminación por aluminio ni ácidez extrema para lograr soportar capas vegetales. El $\%$ de saturación de bases $=80,2 \%$ es un valor alto, ya que esta propiedad se define como la capacidad de cambio catiónico total ocupada por cationes alcalinos como el calcio, magnesio, sodio y potasio. Esta propiedad esta relacionada con el $\mathrm{pH}$ y la fertilidad del suelo, por lo tanto, a mayor $\mathrm{pH}$ y a mayor fertilidad del suelo, mayor será el grado de saturación de bases y mayor será la absorción de cationes.

Respecto a la conductividad eléctria ( $\mathrm{Ce}$ ) $(\mathrm{dS} / \mathrm{cm})=3.0$, lo que indica que es un suelo no salino y no es limitante para el cultivo de alfalfa. El $\%$ de saturación de bases $=80,2 \%$, es un valor alto. Esta propiedad se define como la capacidad de cambio catiónico total oculada por cationes alcalinos como $\mathrm{Ca}, \mathrm{Mg}, \mathrm{Na}$ y K. Esta propiedad esta relacionada con el $\mathrm{pH}$ y la fertilidad del suelo, por tanto, a mayor $\mathrm{pH}$ y a mayor fertilidad del suelo, mayor será el grado de saturación de bases y a mayor grado de saturación de bases mayor es el grado con que los cationes son absorbidos por las plantas.

Sobre el nitrógeno de tejido vegetal analizado, el contenido de nitrógeno es bajo (todos los datos) para niveles que van desde 2,5 a 4,5. Esto supone que los nutrientes nitrogenados del suelo no están siendo asimilados por las especies vegetales analizadas, con excepción del Rumex=2,9; que supera muy debilmente su deficiencia.

\section{CONCLUSIONES}

La fitorremediación no es una solución para todos los suelos contaminados, por ello y antes de ser usada de manera técnica, eficiente y económicamente en procesos de descontaminación-saneamiento, se requiere superar; principalmente, claridad de los mecanismos moleculares, bioquímicos y fisiológicos de las plantas "prospectos" fitorremediadoras. Las especies de plantas encontradas como malezas en los suelos del CAM acumulan en promedio $8.3 \mathrm{mgPb} / \mathrm{kg}$, indicando que estas especies acumulan aproximadamente el $35 \%$ del plomo contenido en los suelos del CAM - Lote-15; unidad de experimen- 
tación. Si los cultivos fuesen de alfalfa, ésta especie acumula plomo; al mismo $\mathrm{pH}=5.4(\mathrm{pH}=5.5$, de experimentación), cerca del $62 \%$ del plomo. Por tanto, si se realiza un cultivo repetido de alfalfa podría considerarse que alfalfa está en capacidad de fito-corregir los suelos de Marengo. El plomo puede ser estabilizado en los tejidos de alfalfa, principalmente en las hojas. Para su disposición final esta especie se debe cosechar y confinar y/o buscar alternativas tales como la briquetación, evitando así que el plomo se encuentre expuesto a los factores ambientales como precipitaciones y viento, evitando que el mismo se disperse en áreas que puedan afectar de forma directa a indirecta a la población (Mosquera, L., y Bernal, C. 2007)

\section{AGRADECIMIENTOS}

Al Instituto Agustín Codazzi por los espacios de laboratorio facilitados y el tipo de análisis realizado. A la Universidad de América, por la consecución de los resultados obtenidos.

\section{REFERENCIAS}

\section{Artículos}

Abollino, O., Aceto, M., Malandrino, M., Mentaste, E., Sarzanini, C. and Barberis, R. (2002). Distribution and Mobility of Metals in Contaminated Sites. Chemometric Investigation of Pollutant Profiles. Environmental Pollution, 119: 177.

Angelova V., Ivanova, R., Delibaltova, V. and Ivanov, $\mathrm{K}$. (2004). Bio-accumulation and distribution of heavy metals in fibre crops (flax, cotton and hemp). Industrial Crops and Products, 19: 197-205.

Arkhipchuk, V.V., Malinovskaya, M.V. and Garanko, N.N. (2000). Cytogenetic study of organic and inorganic toxic substances on Allium cepa, Lactuca sativa, and Hydra attenuata cells. Environmental Toxicology, 15:338-344.

Baker, A.J.M. (1981). Accumulators and excludersstrategies in the response of plants to heavy metals. Journal Plant Nutrition, 3:643-654.

Bernal, BCR. (2008). Diseño de investigaciones experimentales con experimentos estadísticos. Estación Experimental para la Investigación y la Producción a Prueba - Ensayos Aplicados 1, Corporación URUK. Bogotá - Colombia

Brooks, R.R., Lee, J., Reeves, R.D. and Jaffré, T. (1977). Detection of nickeliferous rocks by analysis of herbarium specimens of indicator plants. Journal eochemical Exploration, 7: 49-57.

Chen, Z.S. (1992). Metal contamination of flooded soils, rice plants, and surface waters in Asia. Biogeochemistry of Trace Metals, D.C. Adriano (Ed.). Lewis Publishers Inc., Florida, USA,:pp. 85- 107.

Chen, Z.S., D.Y. Lee, C.F. Lin, S.L. Lo, and W. Y.P. (1996). Contamination of rural and urban soils in Taiwan, Boston, London.
Delgadillo-López, A., González-Ramírez, C., PrietoGarcía, F., Villagomez-lbarra, J., y Acevedo-Sandoval, O. (2011).Fitorremediación: Una alternativa para eliminar la contaminación. Tropical and Subtropical Agroecosystem, 14: 597-612.

Depledge $\mathrm{MH}$, Weeks, JM y Bjerregaard (1994). Heavy metals. In: Hanbook of Ecotoxicology. P Calow (ed). Blackwell Publ. Cambridge. Pp:79-105.

García, I. and Dorronsoro, C. 2005. Contaminación por Metales Pesados. En Tecnología de Suelos. Universidad de Granada. Departamento de Edafología y Química Agrícola. http://edafologia.ugr.es.

Gardea-Torresdey, J. L., Gonzalez, J. H., \& Tiemann, K. J. (1998). Phytofiltration of hazardous cadmium, chromium, lead and zinc ions by biomass of Medicago sativa (alfalfa). Journal Of Hazardous Materials, 5729-39. 
LÍNEA DE INVESTIGACIÓN: REMEDIACIÓN

Guerrero, A. (1999). Cultivos herbáceos extensivos. España:Grupo Mundi-Prensa

lannacone, J., Alvariño, L., Caballero, C. and. Sánchez, J. (2000). Cuatro ensayos ecotoxicológicos para evaluar lindano y clorpirifos. Gayana, 64:139-146.

Matte, T. (2003). Efectos del plomo en la salud y la niñez. Salud Pública México: 45 (2) ISSN: 00363634.

Molina, E. (1998). Enalado para la corrección de la acidez del suelo. ACCS, San José de Costa Rica. 45

Niu, Z., Sun, L., Sun, T., Li, Y., \& Wang, H. (2007). Evaluation of phytoextracting cadmium and lead by sunflower, ricinus, alfalfa and mustard in hydroponic culture. Journal of Environmental Sciences (China), 19(8), 961-967. Retrieved from http://search.proquest. com/docview/289318702?acc ountid $=1460$

Pagnanelli, F., Moscardini, E., Giuliano, V. y Toro, L. (2004). Sequential extraction of heavy metals in river sediments of and abandoned pyrite mining area: pollution detection andaffinity series. Environ. Pollut. 132: 189-201.

Rodríguez O., J.C., Rodríguez, F.H., Lira R.G., De la Cerda, JM., Lara M., J.L. (2006). Capacidad de seis especies vegetales para acumular plomo en suelos Con- taminados. Revista Fitotecnia Mexicana, 29:239-245.

UCLM. Universidad de Castilla - La Mancha. Mineralogía y Geoquímica Ambiental, Tema 4, Prospección Geoquímica. Responsables: Higeras, P; Oyarzun, R. Departamento de Ingeniería Geológica y Minera. España. (Visitado el 19 de Mayo de 2007). http://www. uclm.es/users/higueras/MGA/ Tema04/Prospeccion_geoquimica_2.htm.

Wang, Y.P., Chen, Z.S., Liu, W.S., Wu, T.H., Chaou, C.C., Li, G.C. and Wang, T.T. (1994). Criteria of soil qualityestablishment of heavy metal contents in different categories. Final report of four- year project. Project reports of EPA/ROC. Grant No. EPA-83E3H1-09-02. 54 pp.

\section{Tesis}

Acosta, M., y Montilla, J. Evaluación de la contaminación por cadmio y plomo en agua, suelo, y sedimiento y análisis de impactos ambientales en la subcuenca del rio Balsillas afluente del rio Bogotá (2011). Proyecto de grado para optar el título de Ingeniero Ambiental Sanitario. Programa de Ingeniería Ambiental y Sanitaria. Universidad de la Salle - Bogotá, Colombia. 157 páginas.

Bonilla-Valencia,

SM. (2013). Estudio para tratamientos de biorremediación de suelos contaminados con plomo, utilizando el método de fitorremediación. Grado para obtener el título de Ingeniero Ambiental. Universidad Politécnica Salesiana- Ecuador. 105 páginas.

Mosquera-Narvaez, L., Nuñez-Chicuasuque, L., y Bernal-Bustos, C. (2007). Tesis: Briquetación a escala de laboratorio de residuos vegetales generados en los grandes abastecimientos de mercado en la ciudad de Bogotá. Título para optar el grado de Ingeniero Químico - Facultad de Ingeniería, Departamento de Ingeniería Química. Fundación Universidad de América. Bogotá, Colombia, 122 páginas

Boshell-Rincon, L., y Bernal-Bustos, C. (2008). Propuesta de saneamiento de suelos contaminados con plomo por medio de fitorremediación a escala de laboratorio para el Centro Agropecuario Marengo. Título para optar el grado de Ingeniero Químico - Facultad de Ingeniería, Departamento de Ingeniería Química. Fundación Universidad de América. Bogotá, Colombia, 112 páginas.

Hernández-Zamora, M. (2009). Estudio de la acumulación de plomo y cadmio por Asphodelus fistulosus L., y Brassica juncea L., para fitorremediar Jales. Tesis para obtener el grado de Maestría en Biotecnología, División de Ciencias Biológicas y de la Salud, Universidad Autónoma Metroplitana Iztapalapa. México. 100 páginas. 